\title{
Caficultura, un espacio de encuentro entre la academia y el sector productivo
}

Juan Ignacio Burneo Valdivieso

jiburneo@utpl.edu.ec

Edwin Daniel Capa Mora

edcapa@utpl.edu.ec

Leticia Salomé Jiménez Álvarez

Isjimenez@utpl.edu.ec
Pablo Alejandro Ochoa Cueva

paochoa@utpl.edu.ec

Universidad Técnica Particular

de Loja, Ecuador.
A 100 años de la Reforma Universitaria de 1918 / Intervenciones

RECEPCIÓN: 29/06/17

ACEPTACIÓN FINAL: 24/08/17

\section{Resumen}

Este trabajo aborda los efectos del intercambio de experiencias obtenidas por un grupo de estudiantes, productores y docentes en torno al manejo del cultivo del café. El proyecto surge a partir de la necesidad de generar un acercamiento entre la academia y el sector productivo cafetalero, constituir un espacio de aprendizaje con miras a mejorar la enseñanza académica mediante el afianzamiento de la teoría con la práctica y contribuir a mejorar la producción del sector cafetalero de sur de Ecuador. La articulación academia-sector productivo se planteó como un complemento de la formación tradicional de nuestros estudiantes, y la experiencia de incluirlos en proyectos de vinculación con la sociedad contribuyó a lograr una sólida formación sustentada tanto en los conocimientos como en el apoyo hacia el sector rural. El hecho de agrupar a estudiantes, docentes y productores cafetaleros alrededor de la misma problemática permitió que se dieran soluciones consensuadas a las necesidades particulares de este sector productivo.

Palabras clave

- Educación en servicio

- Docencia-extensión

- Productores cafetaleros

\section{Resumo}

Este artigo aborda os efeitos da troca de experiência adquirida por um grupo de estudantes, agricultores e professores de todo o manejo da cultura do café. O projeto surge da necessidade de criar uma aproximação entre a academia eo setor de produção de café, à procura de um espaço de aprendizagem para melhorar ensino acadêmico fortalecendo a teoria com a prática. Academiaindústria conjunta de produção foi criado como um complemento à formação tradicional dos nossos alunos, a experiência de inclusão de alunos em projetos de ligação com a sociedade que contribuiu para alcançar uma sólida apoiada tanto o conhecimento e apoio à setor rural. Agrupamento dos alunos, professores e produtores de café em torno do mesmo problema, permitiu um consenso em relação às necessidades das soluções do setor produtivo são dadas.

Palavras-chave

- Educação em serviço

- Ensino-extensão

- Produtores de café

\section{Para citación de este artículo}

Burneo Valdivieso, J. I.; Capa Mora, E. D.; Jiménez Álvarez, L. S. y Ochoa Cueva, P. A. (2017). Caficultura, un espacio de encuentro entre la academia y el sector productivo. Revista +E versión en línea, 7(7), 290-295. Santa Fe, Argentina: Ediciones UNL. 


\section{Introducción}

En la actualidad, uno de los retos para las instituciones de educación superior (IES) es el de promover y fortalecer espacios de vinculación entre la academia y el sector productivo. Estos dos sectores, por diversos motivos, se han mantenido apartados el uno de otro en varias regiones de Sudamérica y en la región sur del Ecuador acentuado por su condición de frontera. Allí se evidencia que los sectores productivos se ven relegados en su desarrollo como consecuencia de la centralización en las grandes urbes del país y la falta de recursos que afecta también a las Universidades. De allí la importancia de buscar mecanismos que permitan un acercamiento benéfico para ambos sectores con el fin de propiciar su desarrollo mutuo. Esto implica, sin duda, la participación activa de los involucrados. Las universidades, por su parte, están llamadas a formar profesionales de calidad y a generar los conocimientos que permitan dar soluciones a las problemáticas que demandan los sectores productivos. Desde el ámbito académico, a decir de Flores et al. (1999) debe incentivarse que los profesionales en formación cambien la actitud pasiva del pasado y fomenten la iniciativa y creatividad, además de asumir los conceptos de liderazgo, excelencia académica, valor de conocimientos, ciencia, tecnología e innovación.

El sector productivo, en tanto, es quien permite el desarrollo económico de una región o país, por lo cual está llamado a incorporar a sus líneas estratégicas y de producción nuevos conceptos científicos y tecnológicos y, aún más importante, debe sumar profesionales calificados a sus líneas de administración y producción. Gibbons (1998) menciona que en las próximas décadas las unidades de producción de bienes y servicios serán más automatizadas y requerirán trabajadores calificados, lo que llevará a un desplazamiento paulatino de la mano de obra no calificada. En Ecuador, uno de los sectores productivos de mayor importancia es el cafetalero, de lo cual la Zona de Planificación 7, al sur del país, es un importante referente, ya que dispone de una gran cantidad de variedades y una alta calidad en taza, siendo en esta última década ganador de varios premios a los mejores productores en taza de café (Galindo, 2013). Además, la producción de café en el sur ecuatoriano involucra componentes sociales, económicos y ambientales muy relevantes. La Federación de Asociaciones de Productores Ecológicos de Café del Sur (FAPECAFES) integra a más de 1500 familias de productores y coexiste con otros grupos significativos de productores que no se encuentran asociados, de los cuales no hay información precisa.

A pesar de la importancia del sector, una de las problemáticas que tienen que afrontar los cafetaleros en esta región es la baja productividad que caracteriza a este cultivo, cuestión que se debe principalmente a la existencia de cafetales viejos e improductivos, deficiente calidad de grano, mezcla de variedades y a una incipiente tecnificación que considere, entre otras cosas: la fertilización, uso del agua, prevención y control de enfermedades, podas sanitarias, renovación de cafetales, e incluso lo referente a la poscosecha, acopio y comercialización. A esto se suma la falta de información base que permita la planificación de la actividad cafetalera a nivel de asociaciones y de productores en general (MAG-IICA, 2001; PEFAT, 2014).

Asimismo, el sector tiene otros problemas, como la reducida inversión, la débil organización de los productores y el escaso crédito destinado a los caficultores. A esta situación se agrega el hecho de que las universidades no han fortalecido la vinculación de sus titulaciones afines al sector agrícola con los sectores productivos, específicamente con el cafetalero, ni han desarrollado adecuadamente sus programas de extensión como un sistema para mejorar la calidad de la docencia universitaria y el aprendizaje cooperativo de sus estudiantes para favorecer integración de estos con las necesidades sociales. Cuestiones fundamentales para superar la dicotomía intramuros/extramuros con que nació la extensión (Barrionuevo y Garat, 2016).

A estos efectos, con relación a la situación actual de la producción cafetalera del Ecuador y en particular de la Región 7, la Universidad Técnica Particular de Loja (UTPL), por medio de su titulación de Ingeniería Agropecuaria, con el aporte de entidades externas, como la Fundación Suiza de Cooperación para el Desarrollo Técnico, SWISSCONTACT, desarrolló el proyecto denominado "Impulsar desarrollo de la caficultura del sur del Ecuador a través de la identificación de sus condiciones productivas y socio-económicas, vinculando la participación de la academia en el sector cafetalero" con el fin de promover el desarrollo de la caficultura en el cantón Loja, al sur del Ecuador. Los resultados del proyecto nos permitieron identificar las necesidades emergentes del sector cafetalero con respecto a la problemática expuesta y se evidenció la necesidad de la generación y gestión del conocimiento en forma participativa (academia, productores, entidades de apoyo al sector). Por lo expuesto, el presente estudio tiene como objetivo, analizar los efectos del intercambio de experiencias que se dieron entre la academia y el sector cafetalero sobre el manejo del cultivo del café en el marco del proyecto mencionado.

\section{Conformación del equipo de trabajo}

El objetivo del proyecto surgió a partir de la necesidad de generar espacios que acerquen a la academia con el sector productivo de manera de orientar conocimientos y buscar soluciones a los problemas específicos del sector cafetalero. Esto tanto en lo que respecta a su desarrollo local como a la obtención de modelos de gestión para optimizar los recursos. El proyecto también busca la consolidación de un espacio de aprendizaje con miras a mejorar la correlación docencia-práctica.

Con estos lineamientos, el trabajo aborda una experiencia realizada por un grupo de estudiantes, productores y docentes en torno al 


\section{6}

una de las problemáticas que tienen

que afrontar los cafetaleros en esta región

es la baja productividad que caracteriza

a este cultivo manejo del cultivo del café. Las actividades del proyecto fueron integradas al componente académico de gestión productiva de la titulación de Ingeniería Agropecuaria, en la que la articulación academia-sector productivo se planteó como una instancia complementaria de la formación sistémica de nuestros estudiantes. El equipo de trabajo estuvo conformado por 20 estudiantes del cuarto año de Ingeniería Agropecuaria y 4 docentes relacionados con los campos profesionales de Ingeniería: Alimentos,

Agropecuaria, Agronómica y Ambiental. La misión del equipo fue motivar a los productores cafetaleros y el resto de la comunidad para que sean partícipes de esta propuesta, incentivándolos a actualizar la información de sus fincas para que, posteriormente, pongan en práctica los planes de manejo del cultivo y fertilización de sus parcelas, los que serían elaborados como resultado del trabajo realizado por la universidad en la asociación de caficultores. El trabajo del equipo, sin embargo, no solo contempló los aspectos técnicos inherentes al manejo del cultivo, sino que también implicó aspectos sociales a partir de la vinculación de los actores (productores, docentes y alumnos) y sus instituciones.

\section{Acercamiento hacia la comunidad}

Si bien es necesario distinguir las características propias que tienen las instituciones de educación superior y las del sector productivo, es de importancia, como se ha mencionado, establecer una alianza estratégica que puede traer importantes beneficios para ambos sectores. El primer paso que se debe dar en este sentido es romper tanto el temor de amenaza a la autonomía propia de cada sector como la desconfianza que ambas partes tienen una de otra. Para eso se requiere un compromiso mutuo de acción compartida, en la que cada actor (academia y comunidad) conviertan sus fortalezas en contexto de enseñanza-aprendizaje, en una oportunidad de modificar su entorno y el de su contraparte. Ubicada en la provincia de Loja, al sur del Ecuador, y más concretamente en la parroquia San Pedro de Vilcabamba, se encuentra la Asociación de Productores Ecológicos de Café Especial del Cantón Loja (APECAEL), que cuenta con alrededor de 70 socios caficultores que aportan con su producto para la venta al extranjero.

Se escogió trabajar con APECAEL ya que, de todas las agrupaciones pertenecientes a la Federación de Asociaciones de Productores Ecológicos de Café del Sur, es la que más necesidades y deficiencias presenta. APECAEL es una asociación con una estructura administrativa débil que no lleva registro de los datos de sus socios, no cuenta con un sistema adecuado de control de las entradas y salidas de lotes de producción de café ni con una adecuada planificación del manejo de sus cultivos ni planes de fertilización, características que la hacen vulnerable frente a otras asociaciones de la región que poseen una estructura técnica y administrativa más fortalecida.

La estrategia empleada en este proyecto fue trabajar directamente con los caficultores a través de su Asociación: la planificación del trabajo incluyó una socialización del alcance del proyecto por parte de los docentes de Ingeniería Agropecuaria ante los directivos de la APECAEL, en la cual se establecieron la metodología y las posibles fechas para iniciar el relevamiento de información. Una vez delimitados los aspectos generales, se tomó contacto con cada uno de los caficultores que serían beneficiarios del proyecto, aspecto clave que dio como resultado que los productores cafetaleros mostraran su interés y su voluntad de colaboración al empoderase de las actividades planteadas.

Por otro lado, la planificación del proyecto también contempló las charlas previas con los estudiantes, en las que se les impartieron los lineamientos del trabajo, los deberes y responsabilidades que tendrían, así como todas las indicaciones técnicas sobre las actividades a desarrollar.

Los estudiantes de Ingeniería Agropecuaria, junto con los docentes, realizaron las salidas de campo, las visitas a cada una de las fincas seleccionadas en donde entrevistaron a los caficultores, recorrieron con el productor cada una de las fincas y parcelas para recopilar 
información agronómica, efectuaron encuestas y toma de muestras de suelos. La información obtenida se registró en una ficha de campo previamente diseñada por el equipo de docentes donde constan, entre otros, los datos sobre: número y listado de socios, área y posición geográfica de la parcela, nombre de variedades cultivadas, volumen de producción, caracterización de la parcela (asociaciones de producción, fertilización, riego, podas de renovación o sanitarias, fenología, incidencia de plagas, etcétera). Con la información recopilada se elaboraron los planes de manejo del cultivo y fertilización de las parcelas de cada productor con las recomendaciones de enmienda de los terrenos, las que posteriormente se socializaron con cada uno de los productores cafetaleros beneficiarios del proyecto. La socialización no solamente consistió en la entrega de dichos insumos a los productores sino que fue más allá, con la asistencia personalizada de cada estudiante a cada socio caficultor, permitiendo el acercamiento directo de los estudiantes con los miembros de la comunidad.

\section{Efectos de la interacción entre la academia y el sector productivo \\ Según María Inés Peralta:}

"La extensión universitaria es el espacio de encuentro entre actores universitarios y otros miembros de la comunidad que, a través de la comunicación y el intercambio de conocimientos, promueve un proceso educativo, cultural y científico articulándose con la docencia y la investigación, lo cual posibilita una relación transformadora de la realidad". (2010:s/n)

Concepto con el que concordamos plenamente, ya que el ingrediente especial que constituyó el soporte de nuestro proyecto fue la interacción de alumnos, docentes y productores cafetaleros mediante un trabajo conjunto de intercambio de conocimientos, experiencias y aprendizajes que se enmarcaron en un ambiente netamente práctico y participativo.
La propuesta planteada en el proyecto permitió que el primer elemento de nuestra ecuación, que son los profesionales en formación de la titulación de Ingeniería Agropecuaria, sean participantes activos de una formación académica distinta de la impartida en el sistema tradicional de educación en el aula. Para ello se necesitó convertir esta experiencia en un espacio en el cual se fusionara la teoría con la práctica para, además, poner a prueba los conocimientos y habilidades de cada alumno.

Biolatto et al. mencionan que

"es impostergable sumar alumnos en trabajos de investigaciónextensión de fuerte contenido e impacto social, pues de esta manera se están promoviendo cambios en la forma de pensar y de actuar profesional de todos estos integrantes." (2016:364)

En correspondencia con ese pensamiento, la experiencia de incluir a nuestros estudiantes en este proyecto de vinculación con la sociedad ha contribuido a lograr una sólida formación sustentada tanto en los conocimientos como en el ámbito social. Esta forma de trabajo con la comunidad posibilitó un cambio muy importante en la formación de los alumnos, ya que ellos participaron en un modelo de trabajo no impartido por la propia Universidad. Los estudiantes tuvieron la oportunidad de intercambiar conocimientos y experiencias directamente con los caficultores, los capacitaron y asesoraron en aspectos teóricos y prácticos.

Ferrer y Barrientos definen al aprendizaje como "un cambio, modificación o transformación del comportamiento de un sujeto, de relativa permanencia, que ocurre como resultado de la práctica" (2016:250).

Así, a partir de reflexiones realizadas por los propios estudiantes, observamos que la experiencia de este proyecto les permitió, justamente, modificar o transformar su criterio ante las problemáticas reales que se presentan en el campo laboral. Del mismo modo, pudieron percibir otras formas de aprendizaje que, en muchos de los casos, no se pueden obtener a partir de la enseñanza tradicional. La posibilidad de acceder a las experiencias de vida de la gente y 


\section{G6}

Los beneficios que se obtendrán a largo plazo se verán reflejados en producciones de alta calidad debido a una mejor gestión de las explotaciones y una ampliación en los márgenes de productividad

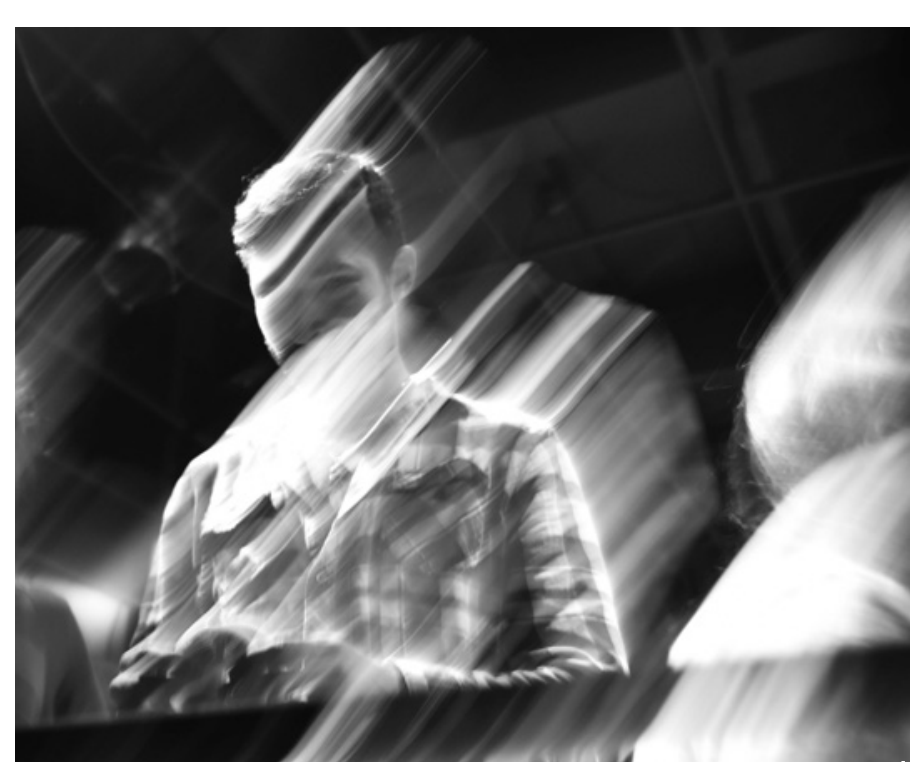

(C) Hugo Pascucci la apertura mostrada por los de productores en cuanto a contar sus experiencias con el manejo del cultivo fueron las piezas clave del aprendizaje que realizaron los alumnos.

Concordamos, por lo tanto, con lo que mencionan Malano et al.:

"A través del modelo de educación experiencial y autogestionaria implementado desde la cátedra Extensión Universitaria, la universidad deja de ser ese espacio concreto de reproducción y transferencia intelectual y formal para transformar lo institucionalizado en una vivencia única e irreproducible". (2016:40)

Existió, además, otro aspecto positivo: la interacción alumnoalumno. El trabajo en campo, en gabinete y en laboratorio se realizó en equipos y se obtuvo una construcción conjunta del conocimiento y un aprendizaje cooperativo, donde emergieron la solidaridad y la confianza en los otros y en sí mismos.

Los productores cafetaleros, como actores extrauniversitarios, fueron, juntamente con los estudiantes, el eje central del proyecto. Tal como Verón et al. mencionan:

"La idea de generar propuestas que superasen el asistencialismo y de trabajar con estos sectores, y no para ellos permite una construcción colectiva y la apropiación de todos los actores del proyecto, convirtiéndonos todos en sujetos de transformación de la realidad emergente". (2016:241)

En ese mismo contexto, en nuestro trabajo, los productores se involucraron por completo ya que fueron parte activa desde la planificación, puesta en marcha y finalización del proyecto, propiciando el protagonismo de la comunidad. Los productores beneficiarios del proyecto y su Asociación se vieron fortalecidos porque recibieron propuestas de posibles mejoras en el manejo de sus cultivos tendientes a incrementar su producción. A su vez, y aún más importante que eso, contaron con un espacio y la oportunidad de compartir sus problemáticas, dudas y preocupaciones con el equipo de trabajo para buscar soluciones en conjunto.

Como un indicador de la apreciación del proyecto y sus resultados, se efectuó una encuesta dirigida a los productores de la Asociación. Uno de los socios entrevistados mencionó que lo más interesante del proyecto fue la relación que mantuvieron con los docentes y estudiantes, calificó a esta actividad como "excelente" y solicitó que a futuro se realicen más vistas y apoyo a la comunidad. Por otra parte, otra de las socias mencionó que los resultados logrados cumplieron con las expectativas que tenían y que el impacto del proyecto en la comunidad fue muy positivo, en tanto que calificó estos dos aspectos como "adecuados". Pidió asimismo que se realicen más análisis de suelos en este sector, ya que enriquecerá la base de datos y con ello mejorarán las planificaciones de fertilización de sus parcelas de café. También era importante recibir las opiniones de los dirigentes de la Asociación. Así, por ejemplo, su administradora consideró "excelentes" los objetivos relacionados con la realidad actual de la comunidad y el cumplimiento de las actividades propuestas y solicitó se extienda a más socios la ayuda brindada en el proyecto. El presidente de la Asociación comentó que el nivel de satisfacción de las necesidades cubiertas por el proyecto fue "adecuado" y pidió que se siga dando apoyo a la comunidad mediante este tipo de proyectos e interacciones entre Universidad y sociedad. 
En el marco del proyecto, los beneficios que se obtendrán a corto plazo van a incidir directamente en la gestión de la Asociación, y a mediano plazo en la calidad de vida de los propios caficultores, quienes se beneficiarán de las mejores decisiones que se tomen. Más a largo plazo, los resultados se verán reflejados en producciones de alta calidad debido a una mejor gestión de las explotaciones y mayor control en las asociaciones y, por lo tanto, una ampliación en los márgenes de productividad.

El tercer elemento involucrado en este proyecto corresponde a los docentes, actores importantes que se constituyen como un puente entre los estudiantes y los productores. La tendencia de enseñanza que manejamos los docentes es la de impartir conceptos que luego relacionaremos con ejemplos. Sin embargo, cuando se trata de llevarlos a la realidad, nos damos cuenta de la complejidad que implica la puesta en práctica de los conceptos teóricos.

Se podría pensar, entonces, que la enseñanza-aprendizaje de manera cooperativa se realiza solamente en la relación alumnoproductor, sin que tenga una influencia en los docentes. Esto no es así: el hecho de que la academia interactúe con los sectores productivos implica no solo que nuestros conocimientos pueden ser mejor aprovechados por la comunidad sino que, también, los docentes nos nutrimos de las experiencias para procurar mejores alternativas y soluciones a las problemáticas del sector productivo. Por eso los docentes estamos llamados a participar activamente en los proyectos de vinculación con la sociedad, tal como fue el caso de esta experiencia; como docentes nos vimos fortalecidos con la puesta en práctica de nuestros conocimientos.

\section{Síntesis de resultados: impacto sobre la comunidad}

Podemos afirmar que, en cuanto al impacto social, la modalidad de haber trabajado directamente con los caficultores a través de las asociaciones ha resultado la manera más adecuada de abordar a la comunidad, ya que implica un contacto directo con la realidad del sector.

La información obtenida durante el proceso fue central para la planificación puesto que, en la mayoría de los casos, la información oficial disponible no es precisa y/o está desactualizada. La valiosa información obtenida en el proyecto permitirá que, a corto y medio plazo, la Asociación beneficiaria alcance un mejor nivel de desarrollo y competitividad.

Otro de los resultados positivos del proyecto fue la generación de una importante consolidación de correlación entre la comunidad y la academia. La posibilidad de integrar a los estudiantes, docentes y productores en torno a la misma problemática permitió que, por el lado de los productores, se convirtieran en sujetos activos y protagonistas de la experiencia, con participación directa de las acciones a la par del equipo de trabajo de la Universidad. Ellos se vieron fortalecidos al recibir las propuestas de modificaciones para mejorar el manejo de sus cultivos, mientras que, desde la academia, se posibilitó a los estudiantes que se enfrentaran con las problemáticas de la comunidad y que fueran artífices de la búsqueda de soluciones.

Podemos concluir, entonces, que de la interacción directa y continua de la academia con el sector productivo dependerá que los profesionales en formación encuentren cabida en las actividades productivas de la región, de manera que contribuyan al crecimiento de la economía nacional. Además, eso supone que ambos sectores asuman su rol de responsabilidad y compromiso frente a la comunidad y en este caso al sector productivo agrícola.

\section{Referencias bibliográficas}

Barrionuevo, A. C. y Garat, J. J. (2016). La formación en extensión como desafío. Reflexiones a partir de la determinación curricular de la extensión agropecuaria en La Plata en los años 60. Revista +E versión digital, 6(6), 64-73. Santa Fe, Argentina: Ediciones UNL.

Biolatto, R.; Vallone, R.; Vallone, C. y Bassi, A. (2016). Proyectos de extensión como promotores de un aprendizaje significativo para alumnos de Ciencias Veterinarias en la Universidad Nacional de Rosario. Revista + E, 6(6), 360-369. Santa Fe, Argentina: Ediciones UNL.

Ferrer, G. y Barrientos, M. (2016). Sinergia entre extensión y docencia en la Facultad de Ciencias Agropecuarias de Universidad Nacional de Córdoba. En Revista + E, 6(6), 248-255. Santa Fe, Argentina: Ediciones UNL.

Flores, A. S.; Jerónimo, G. P. y Herrera, M. C. (1999). Un reto para las universidades latinoamericanas: la vinculación con el sector productivo. Educación y educadores, (3), 33-54.

Galindo, H. (2013). Análisis del mercado español para la exportación de café ecuatoriano de especialidad producido en cariamanga certificaciones orgánicas y de comercio justo. Recuperado de: http://dspace.udla.edu.ec/bitstream/33000/3053/1/UDLA-EC-TLNI-2013-05(S).pdf

Gibbons, M. (1998). Pertinencia de la educación superior en el siglo XXI. Education, Human Development Network, World Bank.

MAG-IICA (Ministerio de Agricultura y Ganadería EC / Instituto Interamericano de Cooperación para la Agricultura CR) (2001). Diagnóstico Cafetero Ecuador. Malano, D.; Sánchez, S. y Elz, R. (2016). ¿Extensión? ¿De qué estamos hablando? En Revista +E versión digital, 6(6), 36-41. Santa Fe, Argentina: Ediciones UNL.

Peralta, M. (2010). La Universidad en la construcción de Políticas Públicas. IV Congreso Nacional de Extensión Universitaria. Mendoza. Disponible en: http:// www.uncuyo.edu.ar/extension/upload/Mar\%C3\%ADa_In\%C3\%A9s_Peralta.pdf (consultado el 20/05/2017).

Plan Económico Financiero de Alternativas Tecnológicas (PEFAT) (2014). PEFAT para el sector cafetalero de la provincia de Loja.

Verón, J.; Hamdan, V. y Natinzon, P. (2016). El programa Autoproducción de Alimentos. Un espacio de intersección entre extensión, investigación y docencia". En Revista + E, 1(6), 240-247. Santa Fe, Argentina: Ediciones UNL. 